Published in Gait \& Posture, 2019, vol. 72 Supp. 1, p. 468-469 which should be cited to refer to this work

DOI: $10.1016 / j . g a i t p o s t .2019 .10 .013$

\title{
LONG-TERM LONGITUDINAL EVOLUTION OF GAIT AFTER TOTAL KNEE ARTHROPLASTY
}

\section{A. Bonnefoy-Mazure (1), M. Attias (1), S. Armand (1), K. Turcot (2), H. Miozzari (1)}

(1) Geneva University Hospitals and Geneva University, Geneva, Switzerland.

(2) Center for Interdisciplinary Research in Rehabilitation and Social Integration and Laval University, Quebec, Canada.

\section{INTRODUCTION}

Total knee arthroplasty (TKA) is the most common surgical procedure for patients with debilitating knee osteoarthritis (OA) [1]. Many studies showed that gait are improved from baseline one year after TKA, but still altered compared to the healthy people [2-5]. However, little is known about impairments and functional limitations of patients several years after TKA [6-7].

\section{RESEARCH QUESTION}

The aim of this study was to investigate the evolution of gait in patients with knee OA before, one year and around seven years after TKA.

\section{METHODS}

Twenty patients (11 females and 9 males) scheduled for TKA were included in this study. Sixteen received a PFC Sigma ${ }^{\circledR}$ TKA (Depuy Orthopaedics, Inc., Warsaw, IN, USA) and 4 a GMK System ${ }^{\circledR}$ TKA (Medacta, Inc, Castel San Pietro, Switzerland). All patients went through a standard rehabilitation program. Each patient had a Clinical Gait Analysis (CGA) before, one year and at a mean of 6.7 (0.6) years (range: 5.5 to 8.2 years) after TKA. The walking speed and three-dimensional knee kinematics for the operated limb were evaluated during a comfortable gait evaluation. Between CGA at one year and 7 years after TKA, three patients received a contralateral TKA, one patient had a revised TKA and one patient received a Total Hip Arthroplasty. Statistical analyses were performed to compare the data before TKA, one year and 7 years after TKA using a repeated-measure ANOVA.

\section{RESULTS}

Seven years after surgery, the patients showed a significant slower walking speed, with similar kinematic parameters, compared to one year after surgery (Table 1 and Figure 1). Knee pain and satisfaction did not change.

\begin{tabular}{|c|c|c|c|c|c|c|}
\hline & \multicolumn{3}{|c|}{ Patients } & \multicolumn{3}{|c|}{$\begin{array}{c}\text { Group comparisons } \\
\text { repeated measure ANOVA (P value) }\end{array}$} \\
\hline & $\begin{array}{c}\text { Before } \\
T K A \\
(\mathrm{n}=20)\end{array}$ & $\begin{array}{c}1 \text { year after } \\
T K A \\
(n=20)\end{array}$ & $\begin{array}{c}7 \text { years } \\
\text { after TKA } \\
(\mathrm{n}=\mathbf{2 0})\end{array}$ & $\begin{array}{l}\text { Before / } \\
1 \text { year }\end{array}$ & $\begin{array}{l}\text { Before / } \\
7 \text { years }\end{array}$ & $\begin{array}{l}\text { One year / } \\
7 \text { years }\end{array}$ \\
\hline \multicolumn{7}{|l|}{$\begin{array}{l}\text { Patients Characteristics } \\
\text { mean (SD) }\end{array}$} \\
\hline Age (years) & $66.6(8.1)$ & $67.6(8.1)$ & $73.1(8.4)$ & 0.914 & 0.039 & 0.096 \\
\hline BMI $\left(\mathrm{kg} / \mathrm{m}^{2}\right)$ & $30.3(5.8)$ & $30.4(6.1)$ & $30.4(6.2)$ & 0.996 & 0.998 & 1.000 \\
\hline \multicolumn{7}{|l|}{$\begin{array}{l}\text { Outcomes - Operated limb } \\
\text { mean (SD) }\end{array}$} \\
\hline Walking speed (m/s) & $1.1(0.2)$ & $1.2(0.2)$ & $1.0(0.1)$ & 0.076 & 0.266 & 0.001 \\
\hline Flexion range - loading response $\left(^{\circ}\right)$ & $5.3(2.0)$ & $5.2(2.3)$ & $6.8(2.5)$ & 1 & 0.076 & 0.076 \\
\hline Range of Motion (ROM) - gait cycle $\left({ }^{\circ}\right)$ & $43.6(8.9)$ & $48.3(7.9)$ & $48.8(7.8)$ & 0.189 & 0.128 & 0.977 \\
\hline $\begin{array}{l}\text { Adduction/Abduction mean absolute value } \\
\text { - stance }\left(^{\circ}\right)\end{array}$ & $6.9(4.8)$ & $3.7(3.2)$ & $5.1(5.5)$ & 0.082 & 0.452 & 0.595 \\
\hline
\end{tabular}

Table 1: Comparisons between patient groups: before TKA, one year months after TKR and 7 years after TKA. Significant differences $(\mathbf{p}<0.05)$ are marked in bold. 


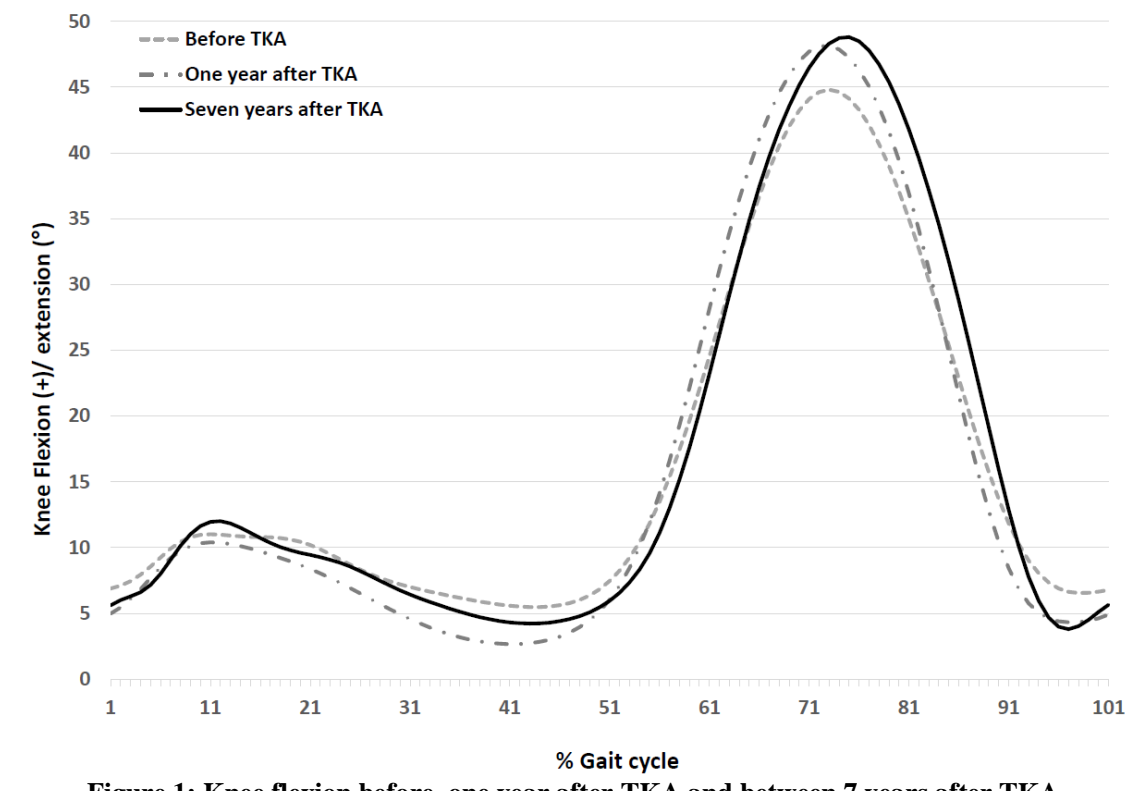

\section{DISCUSSION}

Our results suggest that, at a mean of 7 years after TKA, patients maintain their gait quality and functionality despite a slower gait speed without any change in pain and satisfaction. It is important to note that these results are influenced by the other orthopaedics' surgeries received by the patients between one year and 7 years after TKA.

\section{REFERENCES}

[1] Ethgen et al., JBJSA, 86-A, 963-74.2004. [2] Leffler et al., Gait Posture, 36, 454-60 ; 2012. [3] McClelland et al., Knee, 18, 151-5; 2011. [4] Bonnefoy-Mazure et al.,JA, 32, 793-800.2016 . [5] Turcot et al., JA, 28,1297-1300 ; 2013. [6] Yoshida et al., JOSPT, 42, 1039-1049.2012. [7] Peeters et al., $A O, 88,158-165.2017$. 